\title{
An introduction to mathematical models in sexually transmitted disease epidemiology
}

\section{G P Garnett}

Mathematical models serve a number of roles in understanding sexually transmitted infection epidemiology and control. This article seeks to provide the non-mathematician with a description of their construction and use and presents illustrative examples from sexually transmitted infection epidemiology.

T he epidemiology of infectious diseases has moved beyond identifying aetiological agents and risk factors to a more detailed understanding of the mechanisms controlling the distribution of infections and disease in populations. ${ }^{1}$ Mathematical models provide an explicit framework within which to develop and communicate an understanding of infectious disease transmission dynamics. ${ }^{2}$ Because we can identify the contacts necessary for the spread of sexually transmitted infections (STIs) they provide an interesting subject for mathematical models and substantial progress has been made in model development. ${ }^{3-9}$ However, the language of mathematics can be intimidating for those unused to it. This can often lead to interesting work being ignored, or, more significantly, the uncritical acceptance of the results of models. Often mathematical modellers are responsible, either by describing complex and irrelevant detail, or, alternatively, simply presenting a "black box" without ever explaining the assumptions that are critical to the results derived. In the presentation of mathematical models a balance is required so that sufficient and comprehensible descriptions can move the subject forward under the critical scrutiny of the scientific community. This paper aims to provide an overview of the terms and methods used in mathematical models and a brief illustration of the use of models in sexually transmitted disease (STD) epidemiology.

\section{THE FUNCTIONS OF MATHEMATICAL MODELS}

The process of describing a system like the spread of an infectious disease forces one to recognise the assumptions made, the data available to estimate parameter values, and allows for qualitative or quantitative predictions that can be tested by comparison with experimental or observed patterns. Thus, the central role of creating and analysing mathematical models is to develop our understanding of a system. Once the transmission dynamics of an infectious disease are appropriately described by a model it is possible to evaluate the potential impact of proposed interventions. Models should assist in the identifica- tion of successful interventions, their necessary scale, and the role or ability of new technologies to deliver public health benefits. Often mathematical models are called upon to forecast the future. This has been particularly true for HIV where many models have been used with varying success..$^{10-13}$ It is important to understand the limitations placed on forecasts by the poor quality of available data, uncertainty about parameter values, non-linearities in the system, and chance events.

In sexually transmitted disease epidemiology mathematical models can describe the position of individuals within the network of sexual partnerships via which infections spread allowing a more complete identification of risks for acquiring and transmitting infection..$^{5-9}$ The population patterns of STI incidence can be simulated based upon descriptions of patterns of sexual behaviour and pathogen biology and compared with observed patterns to test our understanding. ${ }^{14-16}$ Subsequently, the consequences of health policy, such as poor access to care delaying STD treatment, or the use of screening for asymptomatic cases, can be calculated. ${ }^{17}$

\section{TERMINOLOGY}

The methods used in modelling reflect both aims and stage of development. Models can be categorised by a number of key criteria.

\section{Compartmental versus distributional}

There are different ways of representing the modelled variables. For example, a group of people with an infection could all be grouped together as a "compartment" of infecteds, $Y$, or we could explore the distribution of severity of disease, $s$, in the population, where $y(s)$ is the number with disease of severity $s$ and the total population of infecteds $Y$ is the sum of those in all the different levels of severity, $s$. A compartmental model of gonorrhoea might divide the population into those susceptible, symptomatically and asymptomatically infected, whereas a distributional model could describe gradations of symptoms, perhaps related to the size of the gonococcal colony or the level of immune response.

\section{Discrete versus continuous}

Change in the model population can take place either as a smooth continuous process or in discrete steps. In the former differential calculus developed to explore changes in one variable with a diminishingly small change in another variable is used, whereas in discrete models, difference equations, which reflect the change over the 
whole time step are used. ${ }^{18}$ The sexual interactions allowing the spread of STIs occurs continually, so most models of STIs are "continuous." However, for ease of calculation some might use discrete time steps. For example, in demography because of the availability of fertility and mortality rates in 5 year age groups the United Nations uses 5 year time steps to project population growth. ${ }^{19}$ To include the impact of STDs on fertility and mortality in such models requires their impact to be apportioned over 5 year periods.

\section{Deterministic versus stochastic}

In a deterministic model events are not subject to chance and two realisations of a model using the same parameters and exact starting conditions will give exactly the same results. However, results can diverge in the case of deterministic chaos because it is impossible to exactly specify starting conditions and the value of variables. There is debate over the role of chaos in measles where small differences in the fraction infected during troughs in incidence grow into larger differences as infection spreads. ${ }^{20}$ Sexually transmitted diseases do not display this boom and bust pattern, probably because the limited chances of spread have led to more stable mechanisms of persistence. In stochastic models chance is taken into account. There are a number of ways to allow the events in a model to be influenced by chance, but the most common and rigorous method is Monte Carlo simulation, where the set of possible next events is defined with a probability attached to each. A random number generator is then used to calculate when the next event will occur and which of the range of possible events it will be. ${ }^{21}$ One of the advantages of stochastic models is that the distribution of expected outcomes following from a given set of parameters can be generated allowing an exploration of the ability of a model to explain observed pattern. With recent increases in the speed of computers stochastic models have become more convenient and should have an important role in understanding the persistence and control of the bacterial STDs when they are at a low prevalence.

\section{Population averages versus individual based simulations}

An important distinction in the modelling of sexually transmitted infections is whether the population is represented in groups with average rates of contact, risk of transmission, and progress through stages of infection or whether individuals and their contacts are explicitly simulated. The latter level of detail provides a powerful tool as it allows microstructures in the dynamic network of sexual partnerships to be included. ${ }^{462}$ However, it carries significant costs in both identifying parameter values and interpreting which measures are significant in generating results. In between the extremes of a homogeneous population and individual based simulation it is possible to divide the population into groups with similar characteristics-for example, of a similar age, sex, or patterns of sexual activity. Thus, the observed heterogeneity in the population can be captured by stratifying the model population. Much of our initial understanding of the epidemiological consequences of the natural history of STDs has been based on studies using population averages, ${ }^{3}$ understanding more detailed sexual network data has required individual based simulation models.?

\section{Linear versus non-linear}

In linear models the state variables are a simple function of one another with very predictable results, whereas non-linear models are more complex. For infectious diseases the transmission term is assumed to be non-linear as it is a function of the interaction between infectious and susceptible individuals. Many health economic models make linear assumptions - that is, treating one more individual reduces the number of cases by one. However, there are knock on effects which depend upon the epidemiological context. For example, in figure lA the impact of a prophylatic vaccine in a homogeneous population (that is, everyone has the same pattern of risk behaviour) is illustrated. If we assume absolute protection, increasing coverage has a linear impact on prevalence, because it simply removes individuals from the at risk population, whereas the impact of a vaccine that reduces susceptibility increases as it moves towards eliminating infection.

\section{Analytical versus numerical solution}

Often for simple models exact mathematical solutions are possible which provide powerful insights into the relation between parameter values and results. Such solutions are often more precise and elegant. The conceptual understanding of the impact of a vaccine shown in figure lA comes from analytical solutions. However, as models increase in complexity it is often necessary to resort to numerical solution. Here specific parameters are entered and the results calculated. The inclusion of sex, age, and sexual activity in the model used to illustrate the impact of a potential HIV vaccine (fig lB) necessitates numerical solution. Standard methods are available which reduce the errors in deriving results. ${ }^{23}$ Sensitivity analysis allows the impact of varying parameters to be
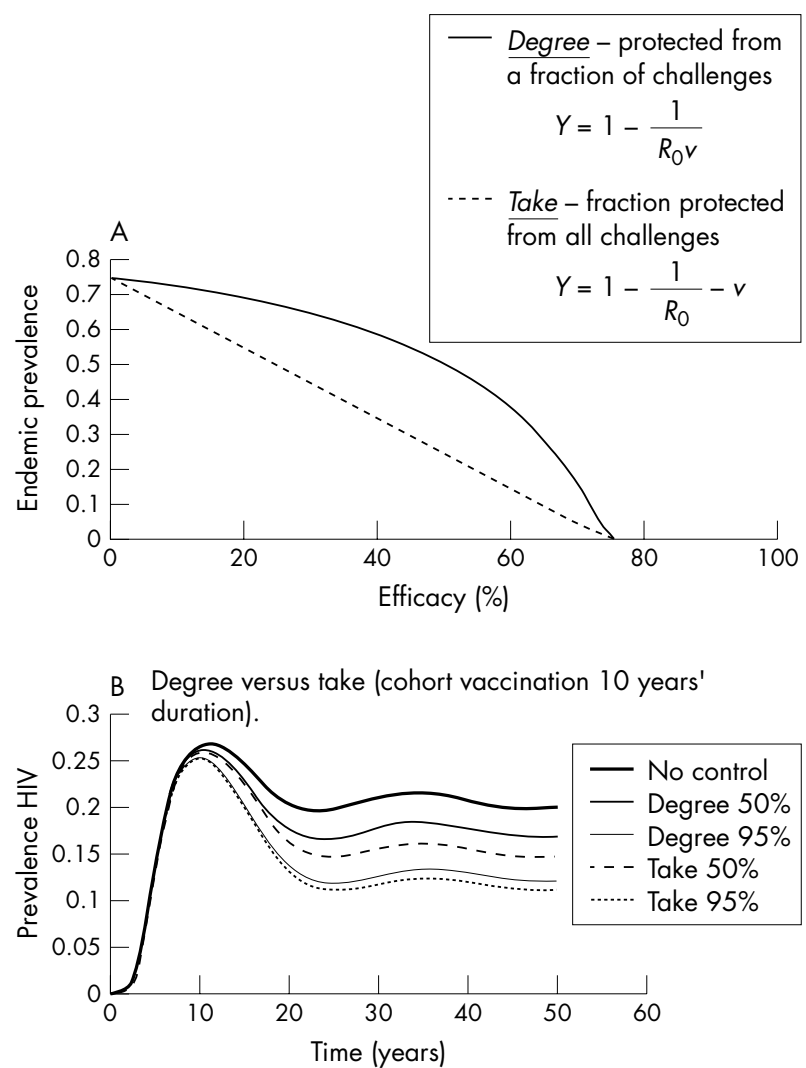

Figure 1 The relation between vaccine efficacy and the prevalence of infection. (A) In a homogeneous population where individuals are fully susceptible, vaccinated, or infected, the vaccine provides two types of protection: "degree" - where for all vaccine recipients efficacy is the fraction of challenges from which they are protected and "take" - where efficacy is the fraction of individuals protected from all challenges. (B) The predicted impact in a model stratified according to sex, age, and sexual activity of an HIV vaccine, with a mean duration of protection of 10 years, introduced after a decade of spread in a generalised HIV epidemic (protection is lost at a constant rate generating an exponentially distributed duration of protection). There are assumed to be two levels of efficacy $150 \%$ and $95 \%$ ) and two types of protection-degree and take. 


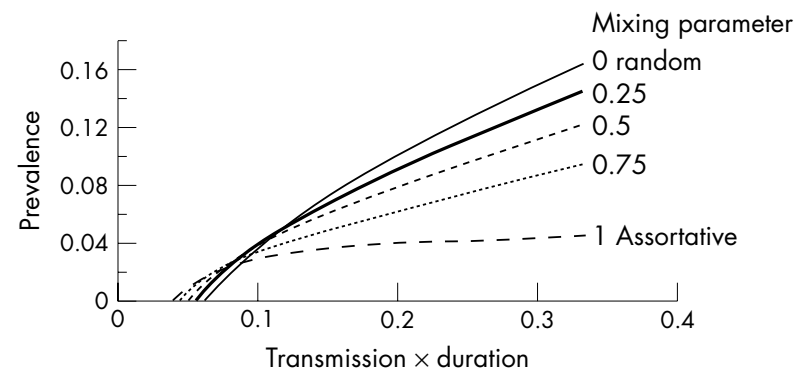

Figure 2 The endemic prevalence a sexually transmitted infection in a heterosexual population stratified according to rates of sexual partner change. The STI is described by a susceptible-infectioussusceptible compartmental model. The endemic (steady state) prevalence is plotted for different biological parameter values with an average rate of partner change of 2 for different patterns of mixing defined on a scale from assortative (like with like) to random according to rates of sexual partner change.

explored, ${ }^{24}$ but such sensitivity analyses are unnecessary for analytical solutions where the influence of changing a parameter is explicit within the model solution.

\section{Sensitivity and uncertainty versus scenario analysis}

In sensitivity analysis combinations of parameter values are chosen at random to explore how the model results are altered by changes in parameters, whereas in uncertainty analysis the impact of potential errors in parameter values is explored. Such approaches often mislead as results depend on model structure as well as parameter values. In scenario analyses illustrative results with chosen sets of parameter values are derived and presented. The numerical solutions describing the impact of a hypothetical HIV vaccine (fig 1B) would be classed as scenario analysis. In figure 2 we illustrate the relation between the duration of infectiousness and the transmission probability and the prevalence of a simple sexually transmitted infection. Each line is a univariate sensitivity analysis and applies only for the set of other parameter values, which remain fixed. The importance of this is illustrated by varying the pattern of mixing which alters the impact of the biological parameters. To explore parameter space in more depth we might sample parameter values at random which was done for a model of trichomoniasis because of the number of poorly estimated parameters. ${ }^{15}$

\section{Quantitative versus qualitative}

One of the paradoxes in modelling infectious diseases is that, despite their quantitative nature, the best that we can often expect is qualitative insights. The difficulty of including all relevant factors, the imprecise measurement of biological and behavioural variables, and the extreme sensitivity of many non-linear systems to small changes in parameter values are frequently insurmountable obstacles to accurate quantitative prediction. None the less, valid qualitative insights can assist policy creation, and in the absence other sources, the evidence of a carefully constructed and defended theory is a better guide than guesswork or intuition. For example, we might be interested in the potential for condoms to control the transmission of HIV. Quantifying the relation between the number of condoms used and the incidence of HIV is confounded by imprecise data on the frequency of sex in at risk partnerships. However, qualitative predictions are possible. The observed heterogeneity between partnerships in the per sex act transmission probability of HIV $^{25}$ indicates that condoms need to be used consistently rather than intermittently within a sexual relationship to have any impact.

The construction of a simple compartmental, continuous, deterministic, non-linear model of an infection in a homogeneous population and its analytical solution is described in the box on the next page.

\section{MODELS AND POLICY}

A key function of models is to predict the consequences of changes such as those caused by interventions. The models provide a tool to translate the changes in patterns of behaviour or biology into an impact on infection and disease. Through exposure to economic models policy makers are predisposed towards their use in decision making and models have the advantage that they can be rapidly deployed when there is insufficient time for field and laboratory studies. Models have significant roles if they generate counterintuitive results (for example, the potential increase in early syphilis that could result from treating latent syphilis ${ }^{1426}$ ) or include the knock on consequences of an intervention not captured in standard health economic analyses (for example, the potential benefits of an HIV vaccine that could reduce the infectiousness of breakthrough infections ${ }^{27}$ ). It is often not necessary to include in full detail the complexity of the system to generate valid results, only sufficient complexity is required. For example, in exploring the elimination of syphilis, stochastic models that represent individual infections are required. In deterministic large population approximations an impossibly tiny fraction of infectious individuals could persist and subsequently reintroduce infection. Validation of models is important when appropriate data are available to test the models. However, convincing explanation of the causes of results and factors that have the potential to invalidate them often have to suffice. The following examples illustrate the use of mathematical models in sexually transmitted disease epidemiology.

\section{Models as a framework for data analysis: a global HIV model}

Mathematical models cannot replace surveillance data, but as data accrue they can provide a framework to analyse and communicate results. Recent work by a UNAIDS reference group has generated a simple and flexible model of the HIV epidemic which provides a framework for the analysis of seroprevalence data from around the globe. ${ }^{28}$ Using maximum likelihood methods the parameters for this model can be estimated from the prevalence data available (fig 3). The initial rate of growth of the epidemic depends largely on a "transmission coefficient", r, where the epidemic peaks depends on the initial fraction at risk, $\mathrm{f}_{0^{\prime}}$ and where the epidemic stabilises depends upon the strength of changes in recruitment to the at risk population in response to AIDS deaths (determined by the value of $\phi$ ). These three values, along with the start time of the epidemic, are estimated from local seroprevalence data, whereas the period between HIV and death, and the birth and death rates are estimated separately and entered. Examples of the use of the model in Uganda and Benin are illustrated (fig 3). The reliability of the resulting HIV curves depends upon the availability and validity of prevalence data. Because such a model is meant to be universally applicable and based on the observed outcome of the epidemic it sheds little light upon the local causes of HIV spread and does not include the detail required to provide insight into interventions.

\section{Understanding interventions: mass antibiotic administration}

Within mathematical models we can explore the relation between the outcome of an intervention and its impact. In developing such models data from a wide range of sources can be combined to define the epidemiological context. In the planning stage of an intervention modelling enables us to evaluate the intervention's potential and set meaningful targets. Additionally, models assist the interpretation of intervention trials, explaining observed results and how they might translate when moved to a different epidemiological context. For example, the expected impact of mass antibiotic administration on the prevalence of trichomoniasis and gonorrhoea is 
compared with syndromic management and illustrated in figure 4 . The coverage and interval between rounds represented is that estimated in the Rakai trial and the results of the determinist model are compared with those from the trial. ${ }^{29}$ The rebound of prevalence following a single round of antibiotic administration is illustrated. This is expected to be faster for gonorrhoea with its high transmission probability than for trichomoniasis. The model is in better agreement for trichomoniasis than gonorrhoea. In the latter case the deterministic model predicts a second rapid rebound after the second round of treatment. However, the low prevalence of infection following treatment will increase the influence of chance delays in the growth of the epidemic. The slower bounce back for trichomoniasis explains the observed reduction in prevalence in the second round of screening. Because of the previous low level of symptom recognition syndromic management is expected to have very little impact on the prevalence of infection. The observed impact indicates either a background trend of decreased STD prevalence, perhaps associated with AIDS mortality and HIV education

\section{The construction of a simple mathematical model}

The model described here is a representation of an infection that causes no acquired immunity. The flow diagram is a means of illustrating the model population, which is compartmentalised into two categories or "state variables" - susceptible or infected.

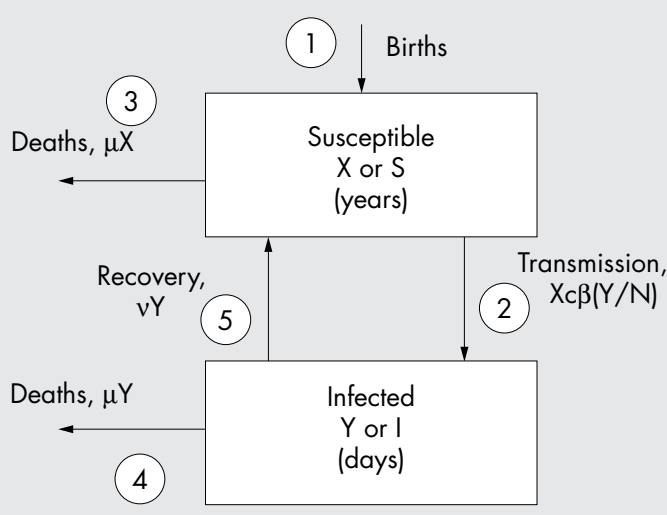

Total population $\mathrm{N}=\mathrm{X}+\mathrm{Y}$
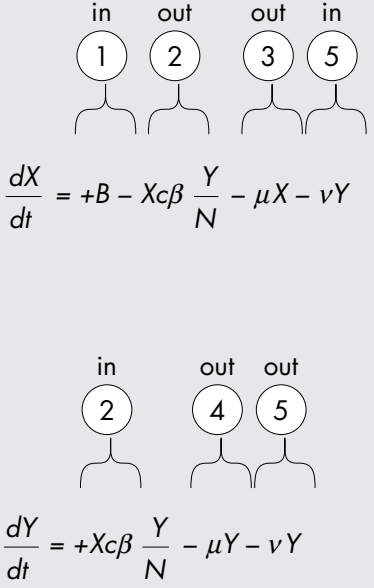

$\frac{d N}{d t}=+B-\mu X-\mu Y$

$d X / d t=$ rate of change in $X$ with respect to time.

People can be in one of these two states, which are represented by boxes. The movement of people in the population is represented by arrows. Such a flow diagram is simply turned into a set of ordinary differential equations. An ordinary differential equation represents the instantaneous rate of change in a state variable with respect to another variable, in this case time. We have an equation for each of the two state variables, $d X / d t$, for the rate of change in susceptible numbers with respect to time, and $d Y / d t$, for those infectious. The flows shown as arrows are calculated using the terms on the right hand side of the equations: a flow out of a box is taken away from a state variable and is a negative term, whereas a flow into a box is added to the state variable, and is a positive term. In the illustration the flows are numbered to show how the terms in the two equations correspond to the arrows in the flow diagram. To derive the terms we need to make assumptions about the flows - for example, initial entry into the susceptible population is by births, termed $B$ in our equation. This could be a number that is consistently recruited each year regardless of the population size. Alternatively, it could be a function of the population size, $N$, and the birth rate for the average individual in the population, $r$, such that $B=r N$. To maintain a constant population size we assume the birth rate equals the death rate, $B=\mu N$. The critical assumptions made about our infection above are that an average susceptible $X$ makes $c$ contacts per unit time; that the fraction of these contacts that are infectious is simply the proportion of the total population infected, $Y / N$ and that there is a chance $\beta$ of transmission on each contact between an infected and a susceptible individual. Thus, the instantaneous total incidence of infection is $X c \beta(Y / N)$ and the instantaneous incidence per susceptible (often described as the force of infection, $\lambda$ ) is $c \beta(Y / N)$. The recovery rate in the above equations is assumed to occur at a constant per infected rate $v$, irrespective of how long someone has been infected. This assumes that a cohort of infecteds will decay exponentially with an average duration of infection given by $1 /(v+\mu)$.

This simple model can be solved analytically, with the equilibrium values being those where the two rates of change are zero. One equilibrium, the disease free state, occurs when there are no infections. The other equilibrium, the endemic steady state, occurs when each new infection causes one more new infection. The value of the basic reproductive number $R_{0}$, which is given by $R_{0}=c \beta /(v+\mu)$, determines which equilibrium is stable. When it is less that one the disease free state is stable alternatively the endemic steady state is stable when it is above one. It should be noted that the basic reproductive number holds for the disease free boundary, as prevalence increases exposures are wasted on those already infected. The reproductive number at time $t$, depends on the basic reproductive number and the proportion of the population susceptible: $R_{t}=R_{0}$ $(X / N)$. At the steady state its value equals one, and because the proportion infected is $1-(X / N)$ we can re-arrange the equation to derive the endemic prevalence $Y / N=1-\left(1 / R_{0}\right)$. 


\section{ANC attendees in Uganda}

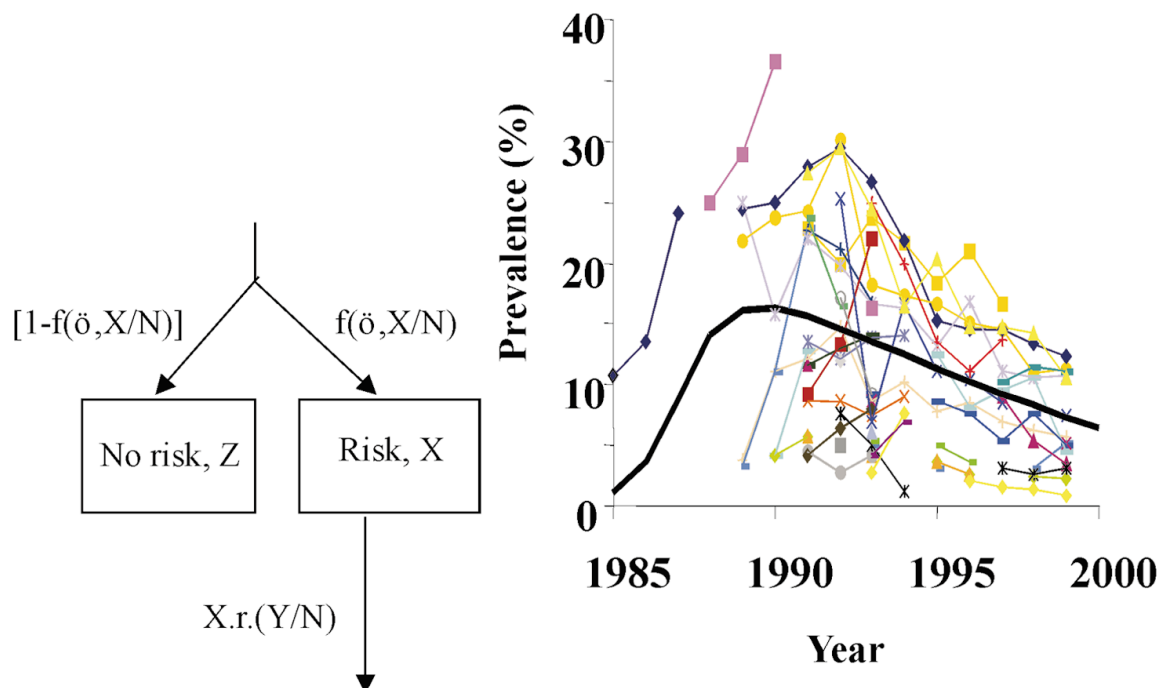

ANC attendees in Benin, West Africa

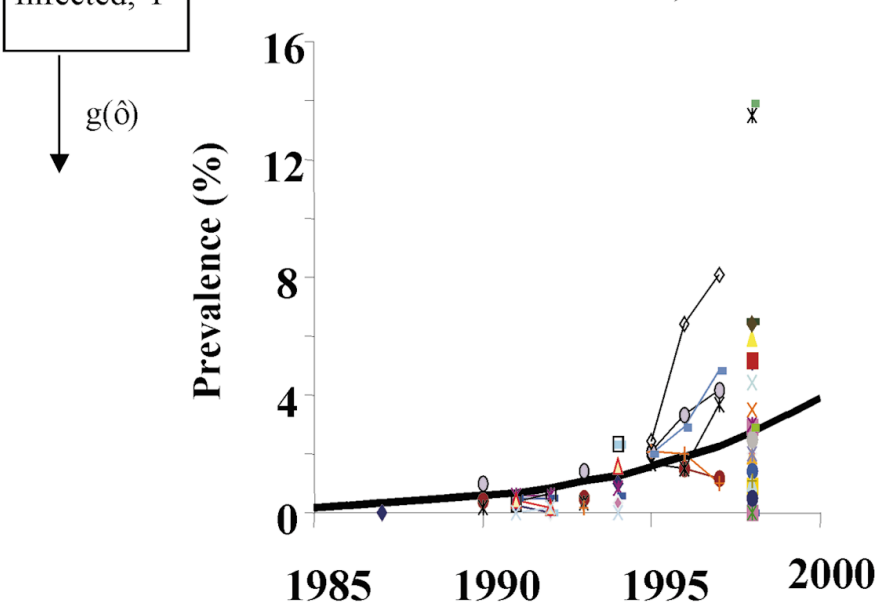

Figure 3 A simple model describing the shape of the HIV epidemic with fits to sentinel HIV surveillance data. (Left) Flow diagram illustrating the modelled population divided into three categories: not at risk; susceptible; and HIV infected. Initially a fraction $f_{0}$ is at risk, but the fraction recruited into the "at risk" class is a function of the proportion of the existing population at risk. This allows the prevalence to stay high or fall precipitously in response to HIV associated mortality. The progress from HIV infection to death is assumed to be Weibull distributed. (Top right) Antenatal clinic (ANC) HIV prevalence data from Uganda with the best fit of the model (solid line) to the observed data. (Bottom right) ANC data from Benin with best fit of model to data. ${ }^{28}$ messages, or that exposure to the trial in the control population had an influence on other epidemiological parametersfor example, recognition of symptoms or condom use. In retrospect, more rapid follow up and administration of antibiotics would have enhanced gonorrhoea control within this trial, but may not have been practical. Model results suggest that the main role of presumptive therapy should be more frequent use in those with a high risk of acquiring and transmitting infection.

\section{The impact of new technology: low efficacy HIV vaccines}

The above conclusions are the more believable because they agree with our intuition (admittedly with the great advantage of hindsight). However, results may not always agree with intuition. For example, we are very used to the high efficacy of vaccines against simple viral infections-smallpox, measles, mumps, rubella, etc- which rely on rapid replication and further transmission to new susceptibles. It might be that the sexually transmitted infections prove a greater challenge with vaccines of lower efficacy. The intuition born of experience with earlier vaccines might lead us to reject such products, but model results demonstrate that a low efficacy HIV vaccine could have a substantial epidemiological impact. ${ }^{30-32}$ However, this should be interpreted with caution. Further analysis of the same model illustrates that a short duration of protection could be a more serious failing (fig 1B). It is important to note that in this model an individual with many sexual partners maintains a high risk over a long period, increasing the significance of the duration of protection from a vaccine. A more detailed analysis of patterns of risk, delivery schedules, and vaccine duration is required to fully understand the potential of particular hypothetical HIV vaccine properties.

\section{GOALS FOR FUTURE MODEL DEVELOPMENT}

Mathematical models of sexually transmitted infections have become more common and more sophisticated, but there is more to be done to demonstrate their worth. Theoretically, understanding the behaviour of infections within the dynamic network of sexual partnerships is a great challenge, particularly since the quality of data on network structures will always be limited and biased. An improved understanding of the pattern of infectiousness within and between infections is another important area where data are sparse and models can have an important role. ${ }^{25}$ In the field of interventions the influence of epidemiological context needs to be explored in more detail, ${ }^{33}$ but also the connection between the input into 

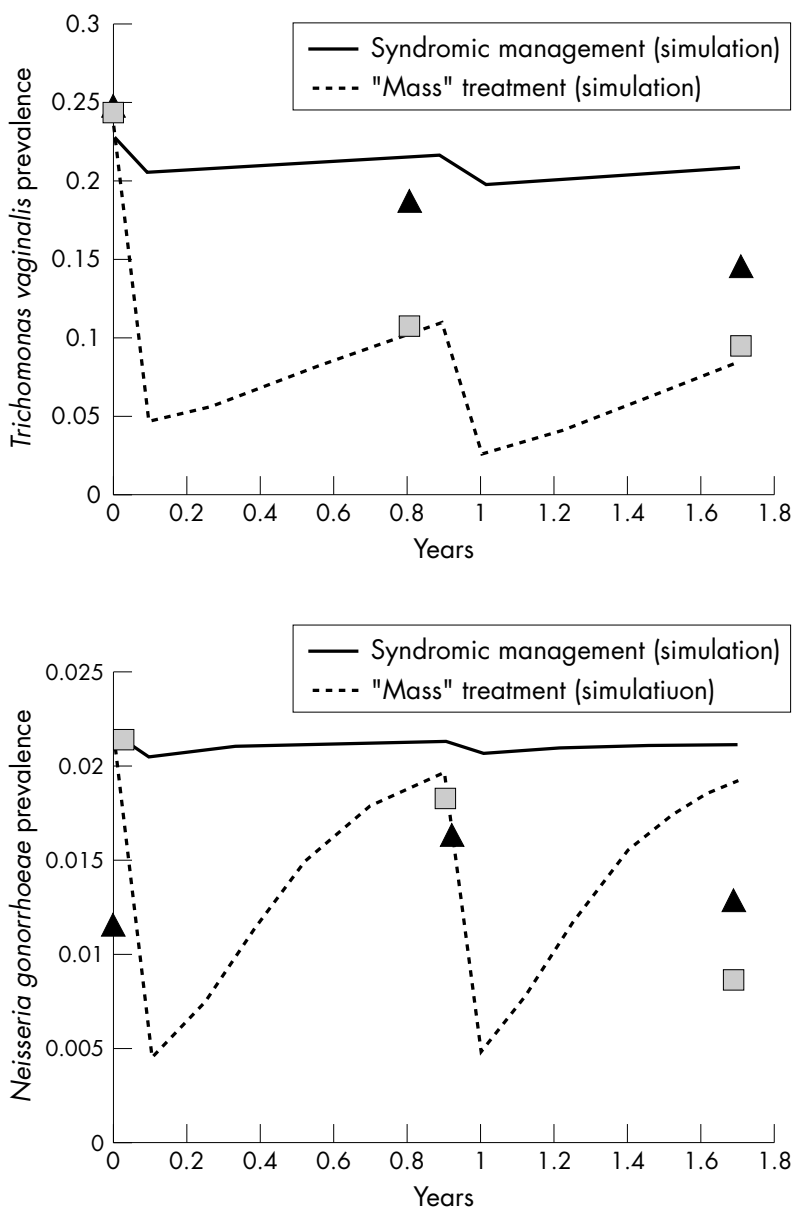

Figure 4 Deterministic model simulations representing the impact of STD syndromic management and mass antibiotic administration with comparisons to their impact in the Rakai trial. ${ }^{29}$ Detailed description of the models and the estimation of parameters are described elsewhere. ${ }^{16}{ }^{17}$ (A) Trichomoniasis treatment; (B) gonorrhoea treatment. Squares, Rakai intervention communities; triangles, Rakai control communities.

interventions and the changing parameter values in mathematical models needs to be better described. Modelling should be an iterative process, with a dialogue between theoreticians, experimentalists, field workers, and policymakers. Models can have an important advocacy role, particularly when they have an attractive user interface, and modelling tools are likely to become increasingly available as user friendly software is developed. Hand in hand with this there is a responsibility not to mislead, which involves the education of the end user about what can and cannot be delivered by mathematical models and the confidence that we can place in our theoretical understanding.

\section{ACKNOWLEDGEMENTS}

Thanks to the Royal Society, the Medical Research Council, and the Wellcome Trust for funding and Dr John Williams and Dr Nick Grassly for help with examples.

\section{REFERENCES}

1 Halloren ME. Concepts of infectious disease epidemiology. In: Rothman KJ, Greenland S, eds. Modern epidemiology. 2nd ed. Philadelphia: Lippincott-Raven, 1998:529-54.
2 Anderson RM, May RM. Infectious diseases of humans: dynamics and control. Oxford: Oxford University Press, 1991.

3 Anderson RM, Garnett GP. Mathematical models of the transmission and control of sexually transmitted diseases. Sex Transm Dis 2000;27:636-43.

4 Ghani AC, Swinton J, Garnett GP. The role of sexual partner networks in the epidemiology of gonorrhoea. Sex Transm Dis 1997;24:45-56

5 Morris $M$, Kretzschmar $M$. Concurrent partnerships and the spread of HIV. AIDS 1997;11:641-8.

6 Kretschmar M, van Duynhoven YTHP, Severijen AJ. Modeling prevention strategies for gonorrhea and chlamydia using stochastic network simulations. Am J Epidemiol 1996;144:306-17.

7 Ghani AC, Garnett GP. Risks of acquiring and transmitting STDs in sexual partner networks. Sex Transm Dis 2000;27:579-87.

8 Ferguson NM, Garnett GP. More realistic models of bacterial STD transmission dynamics:sexual partnership networks, pair models and moment closure Sex Transm Dis 2000/27:600-9.

9 Koopman J, Chick SE, Riolo CS, et al. Modeling contact networks and infection transmission in geographic and social space using Germs. Sex Transm Dis 2000;27:617-26.

10 Anderson RM, May RM, Mclean AR. Possible demographic consequences of AIDS in developing countries. Nature 1988:332:228-34

11 Bongaarts J. A model of the spread of HIV infection and the demographic impact of AIDS. Statistics in Medicine 1989;8:103-20.

12 Chin J, Lwanga SK Estimation and projection of adult AIDS cases: a simple epidemiological model. Bull World Health Organ 1991;69:399-406

13 Report of a working group (Chairman: Day NE), convened by the Director of the Public Health Laboratory Service on behalf of the Chief Medical Officers. The incidence and prevalence of AIDS and the prevalence of other severe HIV disease in England and Wales for 1995 to 1999: projections using data to the end of 1994. CDR Rev $1996 ; 6: R 1-24$

14 Garnett GP, Aral SO, Hoyle DV, et al. The natural history of syphilis:its implications for the transmission dynamics and control of infection. Sex Transm Dis 1997:24:185-200.

15 Bowden FJ, Garnett GP. The epidemiology and control of Trichomonas vaginalis: parameterising and analysing a mathematical model. Sex Transm Inf 2000;76:248-56

16 Garnett GP, Mertz KJ, Finelli L, et al. The transmission dynamics of gonorrhoea: modelling the reported behaviour of infected patients from Newark, New Jersey. Philosophical Transactions of the Royal Society London, B 1999:354:787-97.

17 Garnett GP, Bowden FJ. The epidemiology of bacterial sexually transmitted disease: the problems and opportunities of a cure. Sex Transm Dis 2000;27:588-99.

18 Begon M, Mortimer M. Population ecology: a unified study of animals and plants. 2nd ed. Oxford: Blackwell Scientific, 1986.

19 Preston SH, Heuveline P, Guillot. Demography. Oxford: Blackwell, 2000.

20 Grenfell BT. Chance and chaos in measles dynamics. J Roy Stat Soc B 1992:254:383-98.

21 Rubinstein RY. Simulation and the Monte Carlo method. New York: John Wiley, 1981

22 Potterat JJ, Rothenberg RB, Muth SQ. Network structural dynamics and infectious disease propagation. Int J STD AIDS 1999;10:182-5.

23 Press WH, Teukolsky SA, Vetterling WT, et al. Numerical recipes in C: the art of scientific computing. 2nd ed. Cambridge: Cambridge University Press, 1992

24 Blower SM, Dowlatabadi H. Sensitivity and uncertainty analysis of complex models of disease transmission:an HIV model, as an example. International Statistical Review 1994;62:229-43.

25 Garnett GP, Røttingen JA. Measuring the risk of HIV transmission. AIDS 2001;15:641-3.

26 Korenromp EL, Van Vliet C, Grosskurth H, et al. Model-based evaluation of single-round mass treatment of sexually transmitted disease for HIV control in a rural African population. AIDS 2000;14:573-93.

27 Koopman JS, Little RA Assessing HIV vaccine effects. Am J Epidemiol 1995:142: 113-20.

28 Grassly N, Garnett GP for the UNAIDS Reference Group on HIV epidemiology. Modelling the spread of HIV-1: the basis for the 2001 UNAIDS country-specific estimates and projections of adult HIV prevalence and AIDS mortality. (Preprint held.)

29 Wawer M, Sewankambo N, Serwadda D, et al. Control of sexually transmitted diseases for AIDS prevention in Uganda: a randomised community trial. Lancet 1999;353:525-35.

30 Anderson RM, Swinton J, Garnett GP. Potential impact of low efficacy HIV vaccines in populations with high rates of infection. Proc Roy Soc Lond B 1995;261:147-51.

31 Garnett GP. The influence of behavioural heterogeneity on the population level impact of potential prophylactic HIV-1 vaccines. J Roy Stat Soc, Series A 1998;161:209-25.

32 Anderson RM, Garnett GP. Low-efficacy HIV vaccines: potential for community-based intervention programmes. Lancet 1996;348:1010-13.

33 Grassley N, Garnett GP, Gregson S, et al. The epidemiological context of HIV Interventions. Bull World Health Organ 2001;79:1121-32. 